\title{
Associations between parenting behavior and anxiety in a rodent model and a clinical sample: relationship to peripheral BDNF levels
}

\author{
R Dalle Molle ${ }^{1}$, AK Portella ${ }^{1}$, MZ Goldani ${ }^{1}$, FP Kapczinski ${ }^{2}$, S Leistner-Segala ${ }^{3}$, GA Salum², GG Manfro ${ }^{2}$ and PP Silveira ${ }^{1}$
}

Adverse early-life environment is associated with anxiety-like behaviors and disorders. Brain-derived neurotrophic factor (BDNF) is sensitive to this environment and could be a marker of underlying brain changes. We aimed at evaluating the development of anxiety-like behaviors in a rat model of early adversity, as well as the possible association with BDNF levels. Similar associations were investigated in a sample of adolescent humans. For the rat study, Wistar rat litters were divided into: early-life stress (ELS, limited access to nesting material) and control groups. Maternal behavior was observed from days 1 to 9 of life and, as adults, rats were subjected to behavioral testing and BDNF measurements in plasma, hippocampus, amygdala and periaqueductal gray. For the human study, 129 adolescents were evaluated for anxiety symptoms and perceived parental care. Serum BDNF levels and the Val66Met polymorphism of the BDNF gene were investigated. We found that ELS dams showed more pure contact, that is, contact with low care and high control, toward pups, and their adult offspring demonstrated higher anxietylike behaviors and plasma BDNF. Also the pure contact correlated positively with adult peripheral BDNF. Similarly in humans, there was a positive correlation between maternal overprotection and serum BDNF only in Met carriers. We also found negative correlations between maternal warmth and separation anxiety, social phobia and school phobia. Finally, our translational approach revealed that ELS, mediated through variations in maternal care, is associated with anxiety in both rats and humans and increased peripheral BDNF may be marking these phenomena.

Translational Psychiatry (2012) 2, e195; doi:10.1038/tp.2012.126; published online 20 November 2012

\section{Introduction}

Anxiety disorders contribute significantly to disability, morbidity and mortality. ${ }^{1}$ Early stressful life events, such as childhood abuse and neglect, have been associated with the development of anxiety disorders. ${ }^{2}$ Anxiety is fairly conserved among species and intrinsically linked to stress responses. The nature and timing of early-life events that can induce changes later in life have been assessed in animal models. These animal models show that a stimulus or stressor, such as handling or maternal separation, applied during the first weeks of life can prematurely abort the stress hyporesponsive period. In other words, a stimulus or stressor applied until the end of the second week of life in rats ${ }^{3,4}$ can persistently program the functioning of the hypothalamic-pituitaryadrenal axis in response to stress. ${ }^{5}$ It was shown that neonatal rats exposed to brief periods of manipulation daily for the first weeks of life have an increased ability to cope with stressful stimuli as adults. ${ }^{6-9}$ In contrast, neonatal rats exposed to prolonged periods of daily maternal separation during the first weeks of life display increased anxietylike behaviors and hyperresponsiveness to a stressor as adults. $^{10,11}$
A novel model of dysfunctional maternal nurturing behavior, based on limitation of nesting material in the cage, influences the quality and the quantity of maternal behaviors. This animal model recapitulates an important element of human early-life neglect and abuse. ${ }^{12}$ Pups reared in this model, despite not showing anxiety-like behaviors in the open-field paradigm, show defective cognitive function and high plasma corticosterone levels associated with depletion of corticotropin-releasing hormone mRNA stores in the paraventricular nucleus of the hypothalamus. These changes in the hypothalamic-pituitary-adrenal axis persist to adult life. $^{13}$

Within this context of early life stress and anxiety behaviors, brain-derived neurotrophic factor (BDNF) has emerged as a potential biomarker for anxiety and other psychiatric disorders, such as depression and eating disorders. ${ }^{14-16}$ Stressed animals have reduced BDNF expression in the hippocampus, and depressed patients have decreased brain and blood levels of BDNF; antidepressant drugs can prevent or restore these BDNF reductions. ${ }^{17-21}$ BDNF is a growth factor expressed in the central nervous system that is able to cross the blood brain barrier. It influences the proliferation,

\footnotetext{
${ }^{1}$ Núcleo de Estudos da Saúde da Criança e do Adolescente (NESCA), Faculdade de Medicina, Hospital de Clínicas de Porto Alegre, Universidade Federal do Rio Grande do Sul, Porto Alegre, Brazil; ${ }^{2}$ Programa de Pós-Graduação em Ciências Médicas: Psiquiatria, Faculdade de Medicina, Hospital de Clínicas de Porto Alegre, Universidade Federal do Rio Grande do Sul, Porto Alegre, Brazil and ${ }^{3}$ Serviço de Genética Médica, Hospital de Clínicas de Porto Alegre, Porto Alegre, Brazil Correspondence: R Dalle Molle, Programa de Pós-Graduação em Saúde da Criança e do Adolescente - FAMED - Universidade Federal do Rio Grande do Sul, Ramiro Barcelos 2350, Largo Eduardo Zaccaro Faraco, Porto Alegre 90035-903, Brazil.

E-mail: betinha_dm@yahoo.com
}

Keywords: anxiety; BDNF; developmental origins of adult disease; early trauma; maternal care

Received 14 June 2012; revised 11 October 2012; accepted 11 October 2012 
differentiation, morphology and functional activity of neuronal cells, being involved in dendritic growth, synaptic plasticity and long-term potentiation. ${ }^{22}$ BDNF is also known to be required for proper development and survival of dopaminergic, GABAergic, cholinergic and serotonergic neurons. ${ }^{23}$

A single-nucleotide polymorphism in the coding region of the BDNF gene, Val66Met allele (methionine in place of valine at position 66), is associated with a functional alteration in which there is a decrease in intracellular trafficking and activity-dependent secretion of BDNF. ${ }^{24}$ Interestingly, a genetically modified mouse with a variant BDNF Met allele that reproduces the phenotypic hallmarks found in humans exhibits increased anxiety-related behaviors when placed in stressful settings. ${ }^{25}$ This finding suggests a potential association between the physiology of this neurotrophin and the pathophysiology of anxiety disorders. In addition, the Met allele is associated with poorer episodic memory and abnormal hippocampal activation on functional magnetic resonance imaging. ${ }^{24}$

Translational approaches seem to be helpful to manipulate controlled environments while simultaneously testing for clinically significant correlates. Some studies already documented the association between parenting (and parentrelated adversity) and anxiety disorders in humans. ${ }^{26,27}$ However, very few of them aim to investigate biological correlates of such association, that is, to understand the mechanisms that link adversity to anxiety disorders. Given previous associations linking BDNF to both childhood trauma and psychopathology, we investigated its role in explaining such association.

Therefore, the aim of this study was to evaluate the development of anxiety-like behaviors in a rat model of early adversity affecting the quality of maternal care, as well as the possible association with peripheral and central BDNF levels. In addition, in an attempt to establish the relevance of our experimental findings to humans, we investigated similar associations in a sample of adolescents.

\section{Materials and methods}

Rat studies. Pregnant Wistar rats, bred at our animal facility, were single housed in home cages $(40 \times 40 \times 30 \mathrm{~cm})$ with a metal mesh bottom $(1 \times 1 \mathrm{~cm})$ kept $2 \mathrm{~cm}$ apart from a removable metal plate used to collect urine and droppings. During pregnancy the floor was covered with wood chips and dams were maintained in a controlled environment: standard dark/light cycle (lights on between $0900 \mathrm{~h}$ and $1900 \mathrm{~h}$ ), temperature of $22 \pm 2{ }^{\circ} \mathrm{C}$, cage cleaning once a week, and food and water provided ad libitum.

The date of birth was considered as day 0 . On day 2 , dams and pups were randomly allocated to two groups (see below) and kept undisturbed until day 9. Maternal behavior was observed from days 1 through 9 (see details below). On day 10 , dams and pups were removed to Plexiglas home cages $(46 \times 31 \times 16 \mathrm{~cm})$ with a wood chip-covered floor and kept in the same controlled environment cited above. ${ }^{12}$

On postnatal day (PND) 21, pups were weaned, separated by sex into two or three per cage and kept in a controlled environment similar to that described above (except for the light cycle-lights on between 0700 and 1900 h). At the time of cage cleaning, body weight was measured using a scale with $0.01 \mathrm{~g}$ precision (Marte, Canoas, Brazil).

Seventeen litters were used for analysis of maternal behavior, divided in two cohorts. The litter size did not differ between groups (mean \pm s.d.-control: $8.9 \pm 3.68$ and ELS: $10.3 \pm 3.39$, Student's $t$-test, $P=0.409$ ). Nevertheless the litter size was used as a covariate in certain analyzes (see statistical analysis). Forty-four adult males and 51 adult females rats were used in the behavioral tasks initiated at PND60. No more than two pups of the same sex per litter were used for the same experiment. All animal procedures were approved by the Research Ethics Committee of Hospital de Clínicas de Porto Alegre (GPPG/HCPA, project number 09-527). Tasks were performed in climate-controlled behavioral rooms within our animal research facility (Unidade de Experimentação Animal/HCPA).

Early-life stress model. ELS group: as described in Ivy et al., ${ }^{12}$ the ELS group had limited access to nesting material from PND2 to PND9. In the morning of PND2, wood chips were removed without touching the animals and a nesting material consisting of paper towels $\left(\sim 2000 \mathrm{~cm}^{3}\right)$ was provided. This was the only material available for the dam to construct a rudimentary nest area. All litters were left undisturbed and bedding was not changed during PND2-PND9.

Control group: dams and pups were left undisturbed, during PND2-PND9, in a home cage identical to that of the ELS group, but with abundant nesting material available ( $\sim 7200 \mathrm{~cm}^{3}$ of wood chips).

Maternal behavior observations. Maternal behavior of each dam was observed for five 72 min periods per day from PND1 to PND9, by observers who received standardized training in the research group to carry out this task. Briefly, the observations occurred at regular times each day with three periods during the light phase $(1000,1300,1700 \mathrm{~h})$ and two periods during the dark phase $(0700$ and $2000 \mathrm{~h})$ of the light/ dark cycle. Within each observation period, the behavior of each dam was scored every $3 \mathrm{~min}$. The following behaviors were scored: dam on or off the nest, dam licking and grooming (LG) any pup, dam nursing pups in either an arched-back posture or a passive posture in which the dam is lying either on her back or side while nursing the pups. ${ }^{28}$ Behavioral categories were not mutually exclusive, for example, licking and grooming often occurred while the mother was nursing the pups. The behavioral data for each female were analyzed as a percentage of the total number of observations for that female over the entire observation period. The pure contact score was calculated by subtracting 'contact + nursing' and 'contact + LG' from the total contact score. Therefore, 'pure contact' score represents contact without care.

Elevated plus maze test. Adult males and diestrous females were tested $^{29,30}$ between 1000 and $1300 \mathrm{~h}$. In the case of females, estrous cycle phases were determined by vaginal smearing every morning between 0800 and $0900 \mathrm{~h}$, starting a week before the test. On the day of the test, the estrous cycle was confirmed $1 \mathrm{~h}$ before exposure to the apparatus. All animals were transferred to the observational room with red 
light illumination and were allowed to habituate for $30 \mathrm{~min}$. The elevated plus maze apparatus was made of wood and consisted of two opposite open arms $(50 \times 10 \mathrm{~cm})$, two opposite enclosed arms with no roof $(50 \times 10 \times 40 \mathrm{~cm})$ and an open square $(10 \times 10 \mathrm{~cm})$ in the center. The maze was elevated $64 \mathrm{~cm}$ above the floor. The animal was placed in the center of the maze, facing one of the open arms, and remained in the apparatus for $5 \mathrm{~min}$, being filmed during this period. The maze was cleaned after each trial with $70 \%$ ethanol. Films were scored using PlusMZ v1.0 software (available at http://blog.sbnec.org.br/2010/07/softwares-gratuitos -para-analise-do-labirinto-em-cruz-elevado-e-campo-aberto/) by the same observer blind to the neonatal intervention. The number of entries, the time spent in open or enclosed arms and the frequency of head dips were analyzed.

Blood collection, brain dissection and BDNF determination. One day after the last behavioral test, animals at $\sim 120$ days old were decapitated following $4 \mathrm{~h}$ of fasting. Trunk blood was collected into heparinized tubes for BDNF determination. The tubes were centrifuged and plasma was separated and frozen at $-80^{\circ} \mathrm{C}$. The brain was quickly removed and flash frozen in isopentane and stored at $-80^{\circ} \mathrm{C}$.

Plasma BDNF was measured by ELISA, using a commercial kit (ChemiKineTM BDNF Sandwich ELISA Kit, Millipore, Billerica, MA, USA), according to the manufacturer's instructions. The brains were warmed to $-20^{\circ} \mathrm{C}$ and the brain regions (hippocampus, amygdala and periaqueductal gray) were macroscopically dissected through thick sections of $0.25 \mathrm{~cm}$ with the aid of an Atlas. ${ }^{31}$ When identified, the region was carefully isolated and punches of $2 \mathrm{~mm}$ diameter were performed to collect the tissue. For determination of BDNF levels in these structures, samples were homogenized in nuclear extraction buffer I (10 mm HEPES, $10 \mathrm{~mm} \mathrm{KCl,} 0.1 \mathrm{~mm}$ EDTA, 0.1 mm EGTA, pH 7.9) with a protease inhibitor (100:1). Then, a detergent (NP40 1\%) was added and the homogenate was centrifuged at 6000 r.p.m. for $10 \mathrm{~min}$ at $4{ }^{\circ} \mathrm{C}$. The supernatant (cytosolic fraction) was used to determine BDNF levels by ELISA (ChemiKineTM Brain BDNF Sandwich ELISA Kit). The results were normalized by the total protein detected in the supernatant using a commercial kit (Pierce BCA Protein Assay Kit, Thermo Scientific, Rockford, IL, USA), according to the manufacturer's instructions. BDNF values were calculated as $\mathrm{ng} \mathrm{mg}^{-1}$ protein.

Human studies. A total of 2457 adolescents from a community sample were studied during the Multidimensional Evaluation and Treatment of Anxiety in Children and Adolescents - the PROTAIA Project. Details about design, methods and sample characteristics of this project can be found elsewhere. ${ }^{32}$ Briefly, all subjects investigated by the survey that were in the upper quartile of the Screen for Children and Anxiety-Related Emotional Disorders scores (SCARED) $^{33}$ and a $10 \%$ random sample of the three remaining quartiles were selected for further evaluation. This study included a sub-sample of 129 adolescents who completed the entire evaluation, including psychiatric diagnosis and symptoms and genotyping. This protocol, deliberately oversampling for anxiety, was designed to obtain adequate power to investigate the effects of anxiety, as well as internalizing symptoms and disorders.

The Parental Bonding Instrument (PBI) was used to assess subjective perceived parenting in adolescents. It assesses basically three main constructs: warmth, overprotection and authoritarism. ${ }^{34-36}$ Parker $^{34}$, who developed the instrument, found strong negative associations between $\mathrm{PBI}$ care and overprotection scores, concluding that maternal overprotection appears associated with low, rather than with high maternal care. ${ }^{37}$

Human blood samples were collected between 0700 and $1000 \mathrm{~h}$ after a fasting period of 10-12 $\mathrm{h}$. These samples were centrifuged for $5 \mathrm{~min}$ at 4500 r.p.m. and serum was stored at $-80^{\circ} \mathrm{C}$. All BDNF measurements were performed in the same day by sandwich ELISA using monoclonal antibodies specific for BDNF (R\&D Systems, Minneapolis, MN, USA), according to the manufacturer's instructions. DNA was extracted from saliva samples using the DNA 2006 Oragene Kit (Laboratory Protocol for Manual Purification of DNA from $4.0 \mathrm{ml}$ of Oragene (Ottawa, ON, Canada) DNA saliva). BDNF genotyping for Val66Met polymorphism was based on the technique described by Ribases et al. ${ }^{15}$ and divided the subjects into Met carriers and non-Met carriers. The study was approved by the Hospital de Clínicas de Porto Alegre Research Ethics Committee (GPPG/HCPA, project number 08-481).

Statistical analysis. For the rat studies, data were analyzed by one-way analysis of variance (ANOVA), using group as factor (maternal behavior), and two-way ANOVA, using group and sex as factors (weaning weight, body weight gain, plus maze test and BDNF levels). When an effect of litter size was found, this was used as a covariable (maternal behavior, weaning weight and body weight gain). Pearson correlation using maternal care and BDNF levels were performed. Sample size varied in each experiment.

For the human studies, Pearson correlations using the domains of warmth, overprotection and authoritarianism on the PBI and the different SCARED scores (separation anxiety, social phobia, school phobia, generalized anxiety and panic symptoms) were performed. Studies in humans have found that the Val66Met polymorphism can be an important moderator in the association between early stressors and serum BDNF levels. ${ }^{38}$ Therefore, data were split according to the BDNF Val66Met polymorphism and Pearson correlations were performed between the PBI scores and serum BDNF values.

For both rat and human studies, normality tests were performed using Kolmogorov-Smirnov or Shapiro-Wilk tests. Student's t-test was performed to compare two groups regarding a quantitative variable.

Data were analyzed using the Statistical Package for the Social Sciences (SPSS) 18.0 software (SPSS Inc., Chicago, IL, USA). Significance levels for all measures were set at $P<0.05$.

\section{Results}

\section{Rat studies}

Maternal behavior. The percentage of off-nest time was shorter in ELS dams $(\mathrm{F}(1,14)=10.57, P=0.006)$, however, 
they spent more time in the nest without nursing or performing LG, resulting in a longer contact time without care in this group $(\mathrm{F}(1,14)=5.30, P=0.037)$. Moreover, ELS dams spent more time nursing their pups, mainly due to a greater duration of arched-back nursing in the lower, less efficient positions ( 1 and 2$)(F(1,14)=16.67, P=0.001)$. The mean duration of licking and grooming (LG) was similar between the control and ELS groups. However, the standard deviation was 2.7 times smaller in the ELS group (Table 1).

The two cohorts used for these observations were compared with regard to all components of maternal behavior (see experimental procedures - maternal behavior observations) and no statistical differences were found (mean \pm s.d. for LG—cohort 1: $8.38 \pm 2.53$, cohort 2: $7.53 \pm 0.89$, Student's $t$-test, $P=0.60$ ); (mean \pm s.d. for pure contact-cohort 1 : $5.62 \pm 1.68$, cohort 2: $5.72 \pm 3.76$, Student's $t$-test, $P=0.95$ ); data from others components not shown).

Body weight. Two-way ANOVA showed no between-group difference in body weight at weaning, using group and sex as factors and litter size as a covariable (control males $(36.4 \pm 9.07 \mathrm{~g})$, ELS males $(35.9 \pm 7.37 \mathrm{~g})$, control females $(34.6 \pm 8.60 \mathrm{~g})$ and ELS females $(33.4 \pm 7.08 \mathrm{~g})$, $\mathrm{F}(1,90)=0.154, P=0.695)$. At weaning, males were heavier $(\mathrm{F}(1,90)=7.102, P=0.009)$ and pups born in larger litters were lighter $(\mathrm{F}(1,90)=555.11, P<0.0001)$. Body weight gain until PND70 was also similar between groups (control males $(248.8 \pm 19.59)$, ELS males $(245.0 \pm 20.94)$, control females $(165.0 \pm 14.17)$ and ELS females (158.1 \pm 9.52$)$, $\mathrm{F}(1,90)=3.174, P=0.078)$.

Elevated plus maze test. Two-way ANOVA, using group and sex as factors, showed an interaction between group and sex in three variables: time spent in open arms (Figure 1a) $(\mathrm{F}(1,57)=5.75, P=0.02)$, number of entries into open arms (control males 6.2 \pm 2.68 , ELS males $4.6 \pm 2.14$, control females $5.5 \pm 2.17$, ELS females $6.5 \pm 2.53)(F(1,57)=4.02$, $P=0.05$ ) and time spent in enclosed arms (control males $115.0 \pm 26.41 \mathrm{~s}$, ELS males $169.3 \pm 32.20 \mathrm{~s}$, control females $158.1 \pm 36.89 \mathrm{~s}$, ELS females $155.2 \pm 31.79 \mathrm{~s})(\mathrm{F}(1,57)=$ $10.61, P=0.002)$. These results revealed a higher anxietylike behavior in males of the ELS group, which spent less time and entered less frequently in the open arms. There were no effects or interaction when analyzing the number of entries into the enclosed arms (control males 6.8 \pm 1.98 , ELS

Table 1 Comparison between means of variables related to maternal care

\begin{tabular}{|c|c|c|c|}
\hline Variables & Control $(\mathrm{n}=8)$ & $E L S(\mathrm{n}=9)$ & P-value \\
\hline LG & $8.06 \pm 2.02$ & $8.73 \pm 0.73$ & 0.630 \\
\hline Off the nest & $44.01 \pm 5.48$ & $35.24 \pm 2.76$ & $0.006^{*}$ \\
\hline Pure contact ${ }^{\mathrm{a}}$ & $5.66 \pm 2.38$ & $7.43 \pm 2.85$ & $0.037^{*}$ \\
\hline $\begin{array}{l}\text { Arched-back nursing } \\
\text { postures } 1 \text { e } 2\end{array}$ & $32.86 \pm 6.97$ & $40.76 \pm 5.85$ & $0.001^{*}$ \\
\hline $\begin{array}{l}\text { Arched-back nursing } \\
\text { postures } 3 \text { e } 4\end{array}$ & $11.73 \pm 4.70$ & $11.53 \pm 4.96$ & 0.635 \\
\hline
\end{tabular}

Abbreviation: LG, dam licking and grooming.

One-way analysis of variance; litter size used as a covariable; data are expressed as mean \pm s.d.; ${ }^{*} P<0.05$

aPercent of time spent in contact without nursing and without LG, in other words, contact of low quality. males $7.5 \pm 2.04$, control females $7.6 \pm 1.88$, ELS females $8.2 \pm 1.37)$. A group effect was observed in the frequency of head dips (Figure 1b) $(\mathrm{F}(1,57)=7.94, P=0.007)$, showing that ELS was associated with a lower frequency of head dips in both sexes.

Brain-derived neurotrophic factor. Evaluation of plasma BDNF levels by two-way ANOVA, using group and sex as factors, showed that ELS was associated with higher BDNF levels, as ELS animals showed higher BDNF levels (control males $0.17 \pm 0.026$, ELS males $0.24 \pm 0.037$, control females $0.13 \pm 0.019$, ELS females $0.19 \pm 0.026)(F(1,22)=4.79$ $P=0.040$ ). There was no sex effect or interaction (Figure 2).

When investigating whether pure contact was correlated with adult peripheral BDNF, a positive correlation was found between plasma BDNF levels and pure contact $(r=0.512$; $P=0.008$ ). Therefore, the more pure contact was observed during the neonatal period, the higher plasma BDNF levels were found in adult life (Figure $3 a$ ).

The analysis of BDNF levels in the brain structures showed an effect of sex on levels in the hippocampus (control males $1.11 \pm 0.78$, ELS males $1.00 \pm 0.55$, control females $0.38 \pm 0.06$, ELS females $0.41 \pm 0.13) \quad(F(1,15)=9.52$
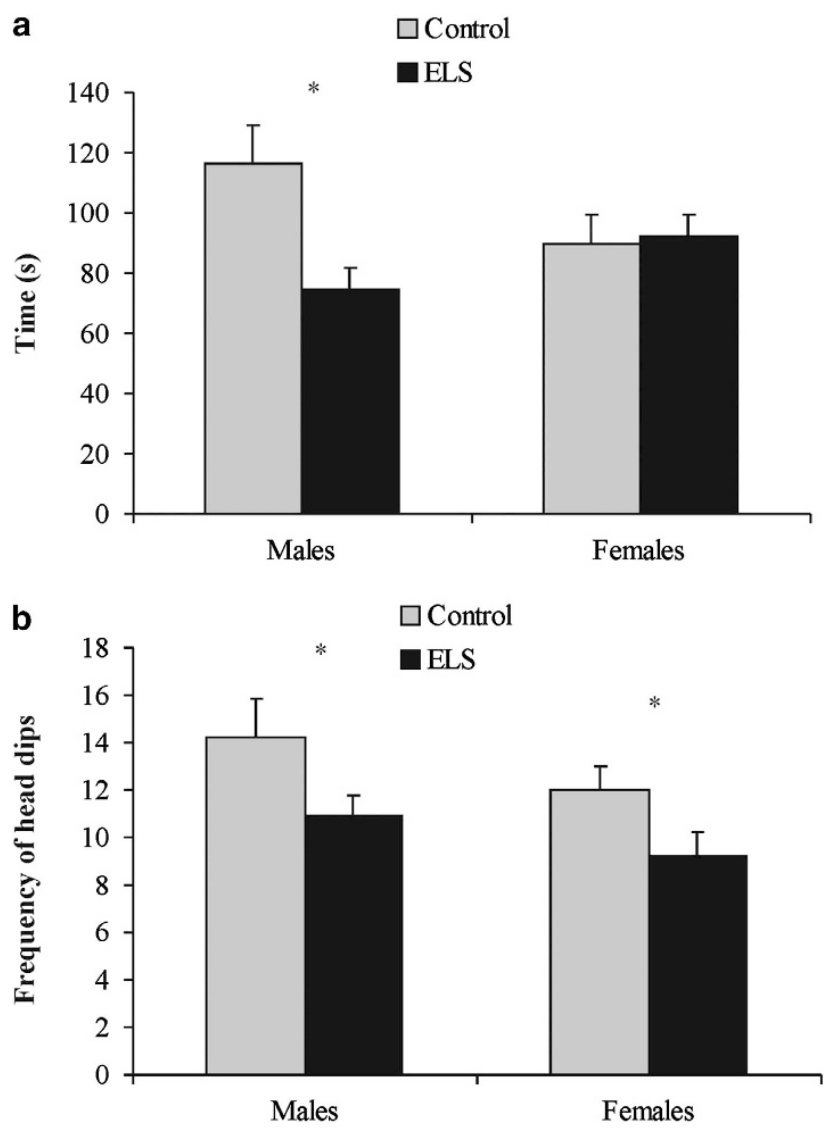

Figure 1 (a) Time spent in open arms and (b) frequency of head dips during the elevated plus maze test in control males $(n=9)$, ELS males $(n=19)$, control females $(n=18)$ and ELS females $(n=15)$. Data expressed as mean \pm s.e.m. Two-way ANOVA showed an interaction between group and sex in the time spent in open arms (a: $\left.{ }^{*} P=0.02\right)$ and a group effect in the frequency of head dips $\left(\mathbf{b}:{ }^{*} P=0.007\right)$. 


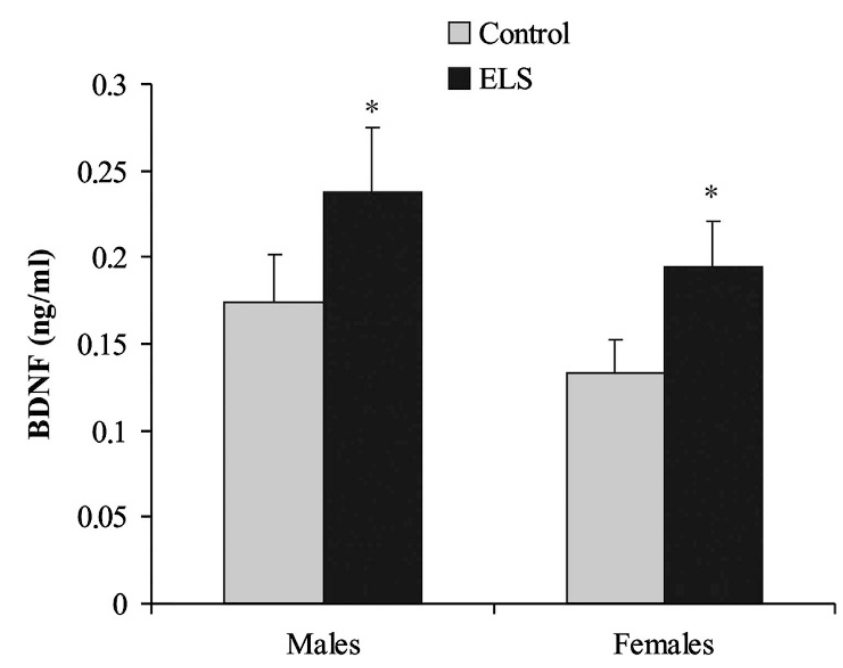

Figure 2 Plasma BDNF levels in control males $(n=6)$, ELS males $(n=7)$ control females $(n=7)$ and ELS females $(n=6)$. Data expressed as mean \pm s.e.m. Two-way ANOVA showed a group effect $\left({ }^{*} P=0.040\right)$.

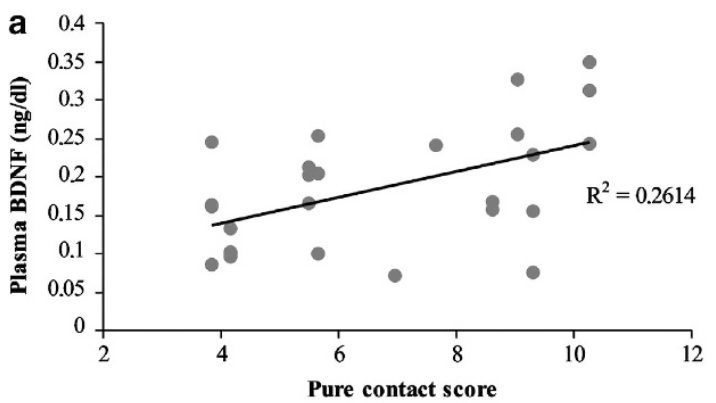

b

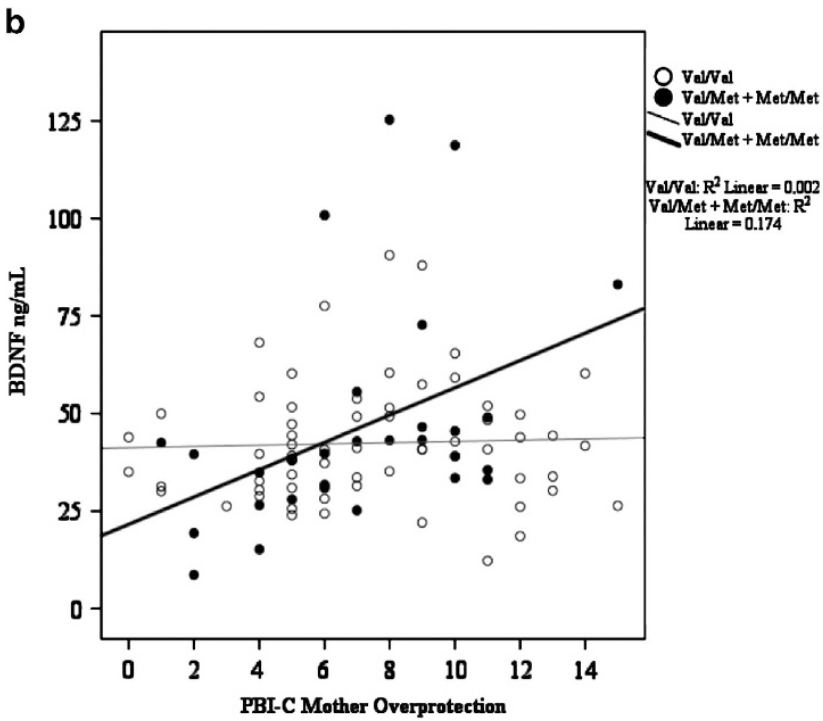

Figure 3 (a) Animal model: correlations between pure contact score and plasma BDNF values $(n=26)$. (b) Humans: correlations between PBI maternal overprotection score and serum BDNF values in Met $(n=30)$ and non-Met carriers $(n=62)$.

$P=0.008$ ) and periaqueductal gray (control males $2.06 \pm 1.04$, ELS males $2.40 \pm 1.45$, control females $0.65 \pm 0.27$, ELS females $0.54 \pm 0.12) \quad(F(1,21)=22.5$,
Table 2 Adolescent sample characteristics $(n=129)$

\begin{tabular}{lccc}
\hline Variables & \multicolumn{2}{c}{ Sex } & P-value \\
\cline { 2 - 3 } & Male $(\mathbf{n}=\mathbf{4 0})$ & Female $(\mathbf{n}=\mathbf{8 9})$ & \\
\hline Age (years) & & $13.62 \pm 2.46$ & 0.064 \\
Postpubertal $^{\text {Menarche (yes) }}$ & $12.77 \pm 2.24$ & $40(44.9 \%)$ & 0.194 \\
Anxiety cases $^{\text {b }}$ & $17(35.0 \%)$ & $57(64.0 \%)$ & - \\
Met carrier $^{c}$ & $13(32.5 \%)$ & $44(49.4 \%)$ & 0.295 \\
& & $31(34.8 \%)$ & 0.572 \\
\hline
\end{tabular}

$\chi^{2} ;{ }^{*} P<0.05$.

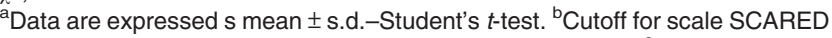
obtained by receiver-operating characteristic curve (ROC). 'BDNF Val66Met polymorphism.

$P<0.001)$, with higher BDNF levels in these structures in males. No differences were seen in the amygdala (control males $0.30 \pm 0.18$, ELS males $2.40 \pm 1.45$, control females $0.65 \pm 0.27$, ELS females $0.54 \pm 0.12$ ). There were no other effects or interactions between variables.

Human studies. There were 89 girls and 40 boys in the sample, but no differences in the mean SCARED score were seen between the two sexes (mean \pm s.d., $32.49 \pm 11.00$ among boys and $33.27 \pm 12.12$ in girls, Student's $t$-test, $P=0.732)$. Other sample characteristics are presented in Table 2.

Negative correlations between maternal warmth on the PBI and the SCARED scores for separation anxiety $(r=-0.222$; $P=0.012)$, social phobia $(r=-0.200 ; P=0.023)$ and school phobia $(r=-0.221 ; P=0.012)$ were found, in which less maternal warmth on $\mathrm{PBI}$ was associated with more anxiety symptoms reported on SCARED. There were no correlations between maternal warmth and generalized anxiety $(P=0.479)$ or panic symptoms $(P=0.575)$. Moreover, there were no other correlations between the other maternal $\mathrm{PBI}$ measurements (overprotection and authoritarianism) and SCARED results (data not shown).

Stratification of data on the basis of absence or presence of the BDNF Val66Met polymorphism revealed a positive correlation between the PBI maternal overprotection score (indicative of a more negative value in the earlier parental interactions) and serum BDNF values $(r=0.417, P=0.022)$ in Met carriers (Val/Met and Met/Met). This association was not found among non-Met carriers $(r=0.039, \quad P=0.765)$ (Figure 3b).

\section{Discussion}

This study showed that in rats limited access to nesting material (ELS paradigm) altered the relationship between dam and pups, and was associated with anxiety and increased peripheral BDNF levels in adult life. Moreover, the degree of pure contact between dams and pups (time spent in contact with pups without LG or nursing, that is, without actually caring for them) correlated positively with plasma BDNF levels in the adult rat offspring. Interestingly, similar associations were found in our sample of adolescent humans, especially for BDNF Met carriers.

Regarding maternal care, the control group showed the well-described normal distribution of the LG score. ${ }^{28}$ On the 
other hand, the model of ELS applied seems to have affected the variance of the LG score, concentrating it at a certain level, and was associated with an increased time spent in lower arched-back postures, which are less efficient in terms of milk letdown. ${ }^{39}$ Classic animal models (such as handling and maternal separation) show that variations in maternal behavior, particularly in the LG scores, are implicated in some aspects of developmental regulation in the rat. Neonatal handling increases LG scores and is associated with reduced behavioral and endocrine stress responses in the adult offspring..$^{6-9}$ On the other hand, separation that deprives pups from active maternal care, induces increased stress and fear responses in the adult offspring. ${ }^{10,11,40}$ The ELS applied in our study wasn't able to alter the mean LG score, but affected its variance. This finding suggests that maternal behavior in the ELS group could be interpreted as 'stereotyped'.

Nevertheless, the most interesting result around the maternal behavior in this animal model seems to be the longest time spent in pure contact, which could suggest a lower quality of maternal care in the ELS dams. These peculiar findings regarding maternal care could be attributed to some differences between our animal model and classic models: first, our study is based on an environmental challenge rather than in maternal separations; second, we studied the full spectrum of LG scores without selecting the dams based on a pre-established LG cutoff, ${ }^{9,41}$ because this selection could be less natural than analyzing the entire cohort.

The ELS paradigm used here replicates a model described for other authors ${ }^{12,13}$ and, because it is based on an environmental change rather than maternal separations, it provides an interesting means of studying the dam/pup relationship without the confounding metabolic consequences of being deprived from maternal milk and warmth. The period chosen for the intervention follows that used in other studies, ${ }^{12}$ which has been shown to be a sensitive period for epigenetic programming of the hypothalamic-pituitary-adrenal axis by variations in maternal care. ${ }^{41,42}$

Interestingly, the ELS applied was able to alter the dam/pup relationship, differently from other classical animal models, yet been associated with the development of adult anxiety in the rat offspring. Similarly, in the human cohort described here low maternal care scores in the $\mathrm{PBI}$ predicted higher levels of anxiety symptoms as measured by the SCARED, similarly to what was originally described by Parker et al. ${ }^{43}$ and others. ${ }^{44}$ Another recent clinical study using the $\mathrm{PBI}$ also showed that, whereas low and high maternal care groups exhibit similarly low responses to acute stress, there are increased levels of depression and anxiety in the low care group. ${ }^{45}$

Furthermore, Parker and Lipscombe ${ }^{37}$ also proposes that maternal overprotection is associated with low, rather than high maternal care, which appears to be consistent with our findings. The increased time spent in 'pure contact' in the ELS group could be interpreted as a behavior related to maternal focus on controlling the nest's physical needs (for example, temperature maintenance), ${ }^{46,47}$ rather than actual caring for pups. It bears noting that this behavior correlates with peripheral measures of BDNF in adulthood, as did PBI overprotection scores in the human cohort of BDNF Met carriers. That is a rigidly controlled type of mothering seems to be associated with increased peripheral BDNF levels and anxiety later in life.

Similar to what was described here, a very recent study showed that decreased maternal care, as assessed by the $\mathrm{PBI}$, correlates with increased harm avoidance and decreased self-directedness in healthy adult subjects and for both personality traits. The partial correlation coefficient was highest in the Met/Met genotype group, suggesting that the BDNF Val66Met polymorphism modulates the effects of parental rearing on these personality traits. ${ }^{48}$ Others have shown that BDNF Met carriers exposed to ELS have elevated neuroticism and higher anxiety, as well as smaller hippocampal and amygdala volumes. ${ }^{49}$ Therefore, as this study reports that the effect of maternal overprotection in the peripheral BDNF levels is evident only in BDNF Met carriers, it is in accordance with the literature, which suggests an increased vulnerability of this gene variant to environmental influences. ${ }^{50}$ Likewise, in rodents, it has been shown that a null mutation in the mouse BDNF gene moderates the long-term effect of maternal care on innate anxiety behavior, ${ }^{51}$ supporting the role of this neurotrophic factor in the development of adult anxiety.

The increased peripheral BDNF levels found in response to early adversity may constitute a way to compensate for a functional deficit in this system, as BDNF has a key role in the maintenance of neuronal integrity. In animal models, however, some studies describe that intra-hippocampal infusions of BDNF induces anxiogenic-like activity. ${ }^{52}$ Interestingly, in our animal model, although ELS was associated with anxiety in the EPM paradigm, there were no differences in central BDNF levels (hippocampus, amygdala and periaqueductal gray). We did not find associations between brain and plasma BDNF levels, so in this animal model peripheral BDNF levels do not seem to directly reflect central BDNF levels, at least in the structures studied. Nevertheless, we cannot rule out a possible pathway dysfunction through the modulation of the number/activity of BDNF receptors or in the induction of the intracellular signaling cascade in our model. Other study using a similar animal model, in which neonatal rats were exposed to either a stressed-abusive mother (maltreatment) or positive caregiving mother (cross-fostered care) only for 30 min daily during the first postnatal week, shows that early maltreatment in rodents produces persisting epigenetic changes in BDNF DNA, altering BDNF gene expression in the adult prefrontal cortex. ${ }^{53}$ In addition, deletion of TrkB in the newborn neuron results in enhanced anxiety-like behavior in adult mice ${ }^{54}$ and mice overexpressing this receptor exhibit attenuated anxietylike behavior. ${ }^{55}$ All these findings on central BDNF/TrkB expression lead us to believe that the differences found in studies about early stress can be attributed to the type of stressors, duration of stress, mouse strain, as well as brain pathway examined.

In both the experimental and the clinical studies reported here, early adversity leading to altered maternal care was associated with increased peripheral BDNF levels. This may be due to an adaptive response associated to the exposure to an acute stressor in a vulnerable period of life, ${ }^{56-58}$ persistently altering baseline BDNF production and increasing 
BDNF levels. It is possible that, as these individuals age or face other stressful situations or diseases during the life course, their resilience will be dampened due to a chronic 'waste' of resources or due to a diminished biological capacity to respond to these insults. Therefore, it would make sense that peripheral BDNF levels are classically decreased in various psychiatric disorders, such as major depression ${ }^{59}$ and bipolar disorder, ${ }^{60}$ when these diseases are associated with childhood trauma.

This paper adds to the growing literature that aims to find biological correlates for the effects of early environmental factors on mental health. Although other authors have studied the relationship between early-life adversity, BDNF and anxiety, ${ }^{51,53}$ this paper offers an interesting opportunity to provide links between clinical and experimental research, as one of the first studies to undertake a translational approach considering the impact of early adversity on anxiety and peripheral BDNF levels in adult life. We recognize this to be a strength of our study, as well as the consistency of the animal model (different cohorts were statistically comparable in the several components of maternal behavior) and the careful to consistently check dams' estrous cycles before the elevated plus maze test, as it is well known that female hormonal changes can interfere with anxiety-like behavior. ${ }^{29}$ Nevertheless, this study also has some limitations. For instance, litter size standardization was not done in the first days of life to avoid effects on maternal care. The variable nutritional status of pups may have influenced study outcomes, however, we were careful to adjust the analysis for litter size in an attempt to minimize this problem. Moreover, the correlations observed between maternal care and anxiety symptoms in humans are significant, though modest. Interestingly, they are mostly consistent with the animal data. In addition, the SCARED is a screening tool for anxiety, not a diagnostic instrument, and we could not take into account potential confounders in the human analysis because of the small sample size. Finally, the human cohort was also oversampled for girls, although there were no sex-related differences in mean SCARED scores. Despite these limitations, we were still able to highlight similarities between the two species, strengthening the validity of our findings. It should be noted that we are still very far from understanding the specificities and complexities of anxiety-like behaviors and a complete inter-species overlap was in no way expected a priori. On the other hand, similar correlates with similar measures provide a promising framework for experimentation in clinically posed research questions.

In conclusion, our study contributes to the body of evidence showing that early trauma that impacts the mother/offspring relationship is associated with anxiety and increased peripheral BDNF levels later in life, both in a rodent model and in a human cohort. The translational approach employed in this study was successful and we propose the rodent model described herein as a useful tool to investigate other mechanisms whereby early adversity altering maternal behavior is linked to adult psychopathology.

\section{Conflict of Interest}

The authors declare no conflict of interest.
Acknowledgements. Financial support from PRONEX 2009, FAPERGS/ CNPq 10/0018.3, Projeto IVAPSA-Impacto das Variações do Ambiente Perinatal sobre a Saúde do Adulto; Brazilian National Council for Technological and Scientific Development (CNPq)-14/2009; Fundo de Incentivo à Pesquisa e Eventos do Hospital de Clínicas de Porto Alegre (FIPE/HCPA); Dalle Molle R had a MSc supporting grant from the Coordination for the Improvement of Higher Education Personnel (CAPES)-Brazil.

1. Balon R. Mood, anxiety, and physical illness: body and mind, or mind and body? Depress Anxiety 2006; 23: 377-387.

2. Weaver IC. Shaping adult phenotypes through early life environments. Birth Defects Res $C$ Embryo Today 2009; 87: 314-326.

3. Guillet R, Michaelson SM. Corticotropin responsiveness in the neonatal rat. Neuroendocrinology 1978; 27: 119-125

4. Sapolsky RM, Meaney MJ. Maturation of the adrenocortical stress response: neuroendocrine control mechanisms and the stress hyporesponsive period. Brain Res 1986; 396: 64-76.

5. Moriceau S, Shionoya K, Jakubs K, Sullivan RM. Early-life stress disrupts attachment learning: the role of amygdala corticosterone, locus ceruleus corticotropin releasing hormone, and olfactory bulb norepinephrine. J Neurosci USA 2009; 29: 15745-15755.

6. Newport DJ, Stowe ZN, Nemeroff CB. Parental depression: animal models of an adverse life event. Am J Psychiatry 2002; 159: 1265-1283.

7. Cirulli F, Berry A, Alleva E. Early disruption of the mother-infant relationship: effects on brain plasticity and implications for psychopathology. Neurosci Biobehav Rev 2003; 27: 73-82.

8. Pryce CR, Feldon J. Long-term neurobehavioural impact of the postnatal environment in rats: manipulations, effects and mediating mechanisms. Neurosci Biobehav Rev 2003; 27: $57-71$.

9. Liu D, Diorio J, Tannenbaum B, Caldji C, Francis D, Freedman A et al. Maternal care, hippocampal glucocorticoid receptors, and hypothalamic-pituitary-adrenal responses to stress. Science 1997; 277: 1659-1662.

10. Plotsky PM, Thrivikraman KV, Nemeroff CB, Caldji C, Sharma S, Meaney MJ. Long-term consequences of neonatal rearing on central corticotropin-releasing factor systems in adult male rat offspring. Neuropsychopharmacology 2005; 30: 2192-2204.

11. Macri S, Chiarotti F, Wurbel H. Maternal separation and maternal care act independently on the development of HPA responses in male rats. Behav Brain Res 2008; 191: 227-234.

12. Ivy AS, Brunson KL, Sandman C, Baram TZ. Dysfunctional nurturing behavior in rat dams with limited access to nesting material: a clinically relevant model for early-life stress. Neuroscience 2008; 154: 1132-1142.

13. Rice CJ, Sandman CA, Lenjavi MR, Baram TZ. A novel mouse model for acute and longlasting consequences of early life stress. Endocrinology 2008; 149: 4892-4900.

14. Lyons WE, Mamounas LA, Ricaurte GA, Coppola V, Reid SW, Bora SH et al. Brain-derived neurotrophic factor-deficient mice develop aggressiveness and hyperphagia in conjunction with brain serotonergic abnormalities. Proc Natl Acad Sci USA 1999; 96: 15239-15244.

15. Ribases M, Gratacos M, Fernandez-Aranda F, Bellodi L, Boni C, Anderluh M et al. Association of BDNF with anorexia, bulimia and age of onset of weight loss in six European populations. Hum Mol Genet 2004; 13: 1205-1212.

16. Hashimoto K, Shimizu E, lyo M. Critical role of brain-derived neurotrophic factor in mood disorders. Brain Res Brain Res Rev 2004; 45: 104-114.

17. Chen B, Dowlatshahi D, MacQueen GM, Wang JF, Young LT. Increased hippocampal BDNF immunoreactivity in subjects treated with antidepressant medication. Biol Psychiatry 2001; 50: 260-265.

18. Karege F, Bondolfi G, Gervasoni N, Schwald M, Aubry JM, Bertschy G. Low brain-derived neurotrophic factor (BDNF) levels in serum of depressed patients probably results from lowered platelet BDNF release unrelated to platelet reactivity. Biol Psychiatry 2005; 57: 1068-1072.

19. Shimizu E, Hashimoto K, Okamura N, Koike K, Komatsu N, Kumakiri C et al. Alterations of serum levels of brain-derived neurotrophic factor (BDNF) in depressed patients with or without antidepressants. Biol Psychiatry 2003; 54: 70-75.

20. Tsankova NM, Berton O, Renthal W, Kumar A, Neve RL, Nestler EJ. Sustained hippocampal chromatin regulation in a mouse model of depression and antidepressant action. Nat Neurosci 2006; 9: 519-525.

21. Warner-Schmidt JL, Duman RS. Hippocampal neurogenesis: opposing effects of stress and antidepressant treatment. Hippocampus 2006; 16: 239-249.

22. Rakofsky JJ, Ressler KJ, Dunlop BW. BDNF function as a potential mediator of bipolar disorder and post-traumatic stress disorder comorbidity. Mol Psychiatry 2012; 17: 22-35.

23. Pillai A. Brain-derived neurotropic factor/TrkB signaling in the pathogenesis and nove pharmacotherapy of schizophrenia. Neurosignals 2008; 16: 183-193.

24. Egan MF, Kojima M, Callicott JH, Goldberg TE, Kolachana BS, Bertolino A et al. The BDNF val66met polymorphism affects activity-dependent secretion of BDNF and human memory and hippocampal function. Cell 2003; 112: 257-269.

25. Chen ZY, Jing D, Bath KG, leraci A, Khan T, Siao CJ et al. Genetic variant BDNF (Val66Met) polymorphism alters anxiety-related behavior. Science 2006; 314: 140-143. 
26. Manfro GG, Otto MW, McArdle ET, Worthington JJ 3rd, Rosenbaum JF, Pollack MH. Relationship of antecedent stressful life events to childhood and family history of anxiety and the course of panic disorder. J Affect Disord 1996; 41: 135-139.

27. Horesh N, Amir M, Kedem P, Goldberger Y, Kotler M. Life events in childhood, adolescence and adulthood and the relationship to panic disorder. Acta Psychiatr Scand 1997; 96: 373-378

28. Champagne FA, Francis DD, Mar A, Meaney MJ. Variations in maternal care in the rat as a mediating influence for the effects of environment on development. Physiol Behav 2003; 79: 359-371.

29. Marcondes FK, Miguel KJ, Melo LL, Spadari-Bratfisch RC. Estrous cycle influences the response of female rats in the elevated plus-maze test. Physiol Behav 2001; 74: 435-440.

30. Severino GS, Fossati IA, Padoin MJ, Gomes CM, Trevizan L, Sanvitto GL et al. Effects of neonatal handling on the behavior and prolactin stress response in male and female rats at various ages and estrous cycle phases of females. Physiol Behav 2004; 81: 489-498.

31. Swanson L. Brain Maps: Structure of the Rat Brain. 2 edn. Elsevier: Amsterdam, Netherlands, 1998.

32. Salum GA, Isolan LR, Bosa VL, Tocchetto AG, Teche SP, Schuch I et al. The multidimensional evaluation and treatment of anxiety in children and adolescents: rationale, design, methods and preliminary findings. Rev Bras Psiquiatr 2011; 33: 181-195.

33. Isolan L, Salum GA, Osowski AT, Amaro E, Manfro GG. Psychometric properties of the Screen for Child Anxiety Related Emotional Disorders (SCARED) in Brazilian children and adolescents. J Anxiety Disord 2011; 25: 741-748.

34. Parker G, Tupling H, Brown LA. Parental Bonding Instrument. Br J Med Psychol 1979; 52: $1-10$.

35. Hauck S, Schestatsky S, Terra L, Knijnik L, Sanchez P, Ceitlin L. Adaptação transcultural para o português brasileiro do Parental Bonding Instrument (PBI). Rev Psiquiatr 2006; 28 : 162-168.

36. Seganfredo AC, Torres M, Salum GA, Blaya C, Acosta J, Eizirik C et al. Gender differences in the associations between childhood trauma and parental bonding in panic disorder. Rev Bras Psiquiatr 2009; 31: 314-321.

37. Parker G, Lipscombe P. Influences of maternal overprotection. Br J Psychiatry 1981; 138 : 303-311.

38. Elzinga BM, Molendijk ML, Oude Voshaar RC, Bus BA, Prickaerts J, Spinhoven P et al. The impact of childhood abuse and recent stress on serum brain-derived neurotrophic factor and the moderating role of BDNF Val66Met. Psychopharmacology 2011; 214: 319-328.

39. Lonstein JS, Simmons DA, Stern JM. Functions of the caudal periaqueductal gray in lactating rats: kyphosis, lordosis, maternal aggression, and fearfulness. Behav Neurosci 1998; 112: 1502-1518.

40. Meaney MJ. Maternal care, gene expression, and the transmission of individual differences in stress reactivity across generations. Annu Rev Neurosci 2001; 24: 1161-1192.

41. Weaver IC, D'Alessio AC, Brown SE, Hellstrom IC, Dymov S, Sharma S et al. The transcription factor nerve growth factor-inducible protein a mediates epigenetic programming: altering epigenetic marks by immediate-early genes. J Neurosci 2007; 27: $1756-1768$

42. Weaver IC, Cervoni N, Champagne FA, D'Alessio AC, Sharma S, Seckl JR et al. Epigenetic programming by maternal behavior. Nat Neurosci 2004; 7: 847-854.

43. Parker G. Reported parental characteristics in relation to trait depression and anxiety levels in a non-clinical group. Aust N Z J Psychiatry 1979; 13: 260-264.

44. Enns MW, Cox BJ, Clara I. Parental bonding and adult psychopathology: results from the US National Comorbidity Survey. Psychol Med 2002; 32: 997-1008.

45. Engert V, Efanov SI, Dedovic K, Duchesne A, Dagher A, Pruessner JC. Perceived early-life maternal care and the cortisol response to repeated psychosocial stress. $J$ Psychiatry Neurosci 2010; 35: 370-377.
46. Stern JM, Johnson SK. Ventral somatosensory determinants of nursing behavior in Norway rats. I. Effects of variations in the quality and quantity of pup stimuli. Physiol Behav 1990; 47: 993-1011.

47. Sokoloff G, Blumberg MS. Thermogenic respiratory, and ultrasonic responses of week-old rats across the transition from moderate to extreme cold exposure. Dev Psychobiol 1997; 30: 181-194.

48. Suzuki A, Matsumoto Y, Shibuya N, Sadahiro R, Kamata M, Goto K et al. The brain-derived neurotrophic factor Val66Met polymorphism modulates the effects of parental rearing on personality traits in healthy subjects. Genes Brain Behav 2011; 10: 385-391.

49. Gatt JM, Nemeroff CB, Dobson-Stone C, Paul RH, Bryant RA, Schofield PR et al. Interactions between BDNF Val66Met polymorphism and early life stress predict brain and arousal pathways to syndromal depression and anxiety. Mol Psychiatry 2009; 14: 681-695.

50. Colzato LS, Van der Does AJ, Kouwenhoven C, Elzinga BM, Hommel B. BDNF Val(66)Met polymorphism is associated with higher anticipatory cortisol stress response, anxiety, and alcohol consumption in healthy adults. Psychoneuroendocrinology 2011; 36: 1562-1569.

51. Carola V, Gross C. BDNF moderates early environmental risk factors for anxiety in mouse. Genes Brain Behav 2010; 9: 379-389.

52. Deltheil T, Tanaka K, Reperant C, Hen R, David DJ, Gardier AM. Synergistic neurochemical and behavioural effects of acute intrahippocampal injection of brain-derived neurotrophic factor and antidepressants in adult mice. Int J Neuropsychopharmacol 2009; 12: 905-915.

53. Roth TL, Lubin FD, Funk AJ, Sweatt JD. Lasting epigenetic influence of early-life adversity on the BDNF gene. Biol Psychiatry 2009; 65: 760-769.

54. Bergami M, Rimondini R, Santi S, Blum R, Gotz M, Canossa M. Deletion of TrkB in adult progenitors alters newborn neuron integration into hippocampal circuits and increases anxiety-like behavior. Proc Natl Acad Sci USA 2008; 105 15570-15575.

55. Koponen E, Voikar V, Riekki R, Saarelainen T, Rauramaa T, Rauvala H et al. Transgenic mice overexpressing the full-length neurotrophin receptor trkB exhibit increased activation of the trkB-PLCgamma pathway, reduced anxiety, and facilitated learning. Mol Cell Neurosci 2004; 26: 166-181.

56. Hauck S, Kapczinski F, Roesler R, de Moura Silveira E Jr, Magalhaes PV, Kruel LR et al. Serum brain-derived neurotrophic factor in patients with trauma psychopathology. Prog Neuropsychopharmacol Biol Psychiatry 2010; 34: 459-462.

57. Grassi-Oliveira R, Ashy M, Stein LM. Psychobiology of childhood maltreatment: effects of allostatic load? Rev Bras Psiquiatr 2008; 30: 60-68

58. Bücker J, Kapczinski F, Kapczinski NS, Cereser KM, Post R, Szobot C et al. (eds)Aumento dos niveis de fator neurotrófico derivado do cérebro e psicopatologia em crianças com trauma quando comparadas a controles. XXVIII Congresso Brasileiro de Psiquiatria. Rev Bras Psiquiatr: Fortaleza, 2010.

59. Grassi-Oliveira R, Stein LM, Lopes RP, Teixeira AL, Bauer ME. Low plasma brain-derived neurotrophic factor and childhood physical neglect are associated with verbal memory impairment in major depression-a preliminary report. Biol Psychiatry 2008; 64: 281-285.

60. Kauer-Sant'Anna M, Tramontina J, Andreazza AC, Cereser K, da Costa S, Santin A et al. Traumatic life events in bipolar disorder: impact on BDNF levels and psychopathology. Bipolar Disord 2007; 9: 128-135.

Translational Psychiatry is an open-access journal published by Nature Publishing Group. This work is licensed under the Creative Commons Attribution-NonCommercial-No Derivative Works 3.0 Unported License. To view a copy of this license, visit http://creativecommons.org/licenses/by-nc-nd/3.0/ 